\title{
EL DESARROLLO DE LA CONCIENCIA PRÁGMÁTICA EN NIÑOS CON SÍNDROME DE ASPERGER
}

Muñoz-Burillo, Lorena; Cascales-Martínez, Antonia

EL DESARROLLO DE LA CONCIENCIA PRÁGMÁTICA EN NIÑOS CON SÍNDROME DE ASPERGER

PANORAMA, vol. 14, núm. 26, 2020

Politécnico Grancolombiano, Colombia

Disponible en: http://www.redalyc.org/articulo.oa?id=343963784010

DOI: https://doi.org/10.15765/pnrm.v14i26.1488

Esta obra está bajo una Licencia Creative Commons Atribución-NoComercial-SinDerivar 4.0 Internacional. 
Artículos de investigación científica y tecnológica

\title{
EL DESARROLLO DE LA CONCIENCIA PRÁGMÁTICA EN NIÑOS CON SÍNDROME DE ASPERGER
}

\author{
THE DEVELOPMENT OF PRACTICAL CONSCIENCE \\ IN CHILDREN WITH ASPERGER SYNDROME
}

O DESENVOLVIMENTO DE UMA CONSCIÊNCIA PRÁTICA NAS CRIANÇAS COM SÍNDROME DE ASPERGER

\author{
Lorena Muñoz-Burillo lore_5595@hotmail.com \\ Universidad de Murcia., España \\ Antonia Cascales-Martínez antonia.cascales@um.es \\ Universidad de Murcia., España
}

PANORAMA, vol. 14, núm. 26, 2020

Politécnico Grancolombiano, Colombia

Recepción: 26 Julio 2018

Aprobación: 05 Febrero 2020

DOI: https://doi.org/10.15765/ pnrm.v14i26.1488

Redalyc: http://www.redalyc.org/ articulo.oa?id=343963784010
Resumen: La conciencia pragmática es la conciencia sobre qué formas del lenguaje son apropiadas al contexto de comunicación, siendo una habilidad para darse cuenta de que uno como hablante u otras personas hacen un uso apropiado o no del lenguaje. En este caso, se analizan las perspectivas del propio niño con asperger, la familia y el terapeuta del propio niño en cuestión sobre la conciencia pragmática que tienen los niños asperger. Para ello, elaboramos un Cuestionario de Conciencia Pragmática (CCP), compuesto por 30 ítems, el cual contestaron ocho niños diagnosticados con SA (síndrome de Asperger), siete madres y un padre de dichos niños y las dos terapeutas que trabajan con ellos. El objetivo propuesto de este trabajo ha sido comparar las perspectivas de la conciencia pragmática de los alumnos con asperger, según ellos mismos, sus familias y los terapeutas a partir del CCP, por un lado, comparando la media de las respuestas en cada uno de los ítems pragmáticos en función de cada bloque y, por otro lado, aplicando un análisis comparativo de las tres perspectivas del CCP. Los resultados revelaron una estructura tridimensional para el CCP y las categorías se agruparon en torno a tres dimensiones pragmáticas: enunciativa, textual e interactiva. El presente trabajo constituye una pequeña aproximación al análisis del comportamiento comunicativo (verbal y no verbal) de niños con SA.

Palabras clave: Conciencia pragmática, síndrome de Asperger (SA), comportamiento comunicativo y contexto de comunicación.

Abstract: The pragmatic consciousness is the awareness of what forms of language are appropriate to the context of communication, with an ability to realize that one speaker or others or make appropriate use of language. In this case, the prospects own child with Asperger's, family and child therapist himself concerned about the pragmatic awareness that have Asperger children are analyzed. To do this, we developed a questionnaire Pragmatic Awareness (CPC), made up of 30 items, which answered: 8 children diagnosed with SA, 7 mothers and one father of these children and two therapists who work with them. The proposed objective of this study was to compare the prospects of pragmatic awareness of students with Asperger, according to themselves, their families and therapists from the CCP. On the one hand, comparing the average of responses on each of the pragmatic items according to each block and on the other hand, applying a comparative analysis of the CCP 3 perspectives. The results showed a threedimensional structure for the CPC and categories are grouped around three pragmatic 
dimensions: declarative, textual and interactive. This work is a small approach to the analysis of communicative behavior (verbal and nonverbal) of children with AS.

Keywords: Pragmatic awareness, Asperger syndrome (AS), communicative behavior and communication context.

Resumo: A consciência pragmática é a consciência de quais as formas de linguagem apropriadas ao contexto da comunicação, sendo uma capacidade de perceber se alguém, como orador ou outras pessoas, faz ou não o uso apropriado da linguagem. Neste caso, as perspectivas da própria criança Asperger, da família, e do terapeuta da criança em questão são discutidas sobre a consciência pragmática que as crianças Asperger têm. Para tal, desenvolvemos um Questionário de Consciência Pragmática (PCC), composto por 30 itens, que foram respondidos por: 8 crianças diagnosticadas com AS, 7 mães e 1 pai destas crianças e os 2 terapeutas que com elas trabalham. O objectivo proposto deste trabalho foi o de comparar as perspectivas da consciência pragmática dos estudantes Asperger, segundo eles próprios, as suas famílias e os terapeutas do PCC. Por um lado, comparar a média das respostas em cada um dos itens pragmáticos de acordo com cada bloco e, por outro lado, aplicar uma análise comparativa das 3 perspectivas do PCC. Os resultados revelaram uma estrutura tridimensional para o CPC e as categorias foram agrupadas em torno de três dimensões pragmáticas: enunciativas, textuais e interactivas.

Palavras-chave: Consciência pragmática, síndrome de Asperger (AS), comportamento comunicativo e contexto de comunicação.

\section{INTRODUCCIÓN}

El trastorno o síndrome de Asperger (SA), fue descrito inicialmente por Hans Asperger en 1944, quien lo identificó como un patrón de comportamiento y habilidades muy específicas, predominante en niños varones, definiéndolo como una 'psicopatía autistica'. Partiendo de esta definición, Wing profundizó más en este síndrome e identificó tres grandes áreas afectadas dentro del SA (Wing \& Gould, 1979; MartínBorreguero, 2005; Vásquez \& del Sol, 2017): trastorno de la relación social, trastorno de la comunicación, incluyendo expresión y comprensión del lenguaje y, finalmente, falta de flexibilidad mental, condicionando al sujeto a un número restringido de conductas y una limitación en las actividades que requieren cierto grado de imaginación.

Según la American Psychiatric Association (APA, 2000), el trastorno de Asperger (TA) es un trastorno generalizado del desarrollo caracterizado por una alteración cualitativa de la interacción social, por la presencia de intereses restrictivos y de comportamientos estereotipados. Los déficits que presentan en interacción social son diversos e incluyen dificultades de comunicación en la comprensión y empleo de las conversaciones lingüísticas y en la interacción social (Whilliams, Keonig, \& Scahill, 2007).

En el DSM-5 (2014), el SA se encuentra dentro del espectro autista, aunque en el DSM-IV (2002), el SA está incluido dentro de los Trastornos Generalizados del Desarrollo (TGD), y es considerado un Trastorno de Espectro Autista (TEA). Los criterios de diagnóstico para hablar del trastorno son:

1. Falta de reciprocidad social y emocional, que se manifiesta en el fracaso en la .onversación, a la hora de compartir intereses, emociones o afectos, etc. 
2. Déficits en la comunicación verbal y no verbal, están poco integrados en la interacción social; a título ilustrativo, destaca la falta de contacto visual, lenguaje corporal como gestos, falta de expresiones faciales, etc.

3. Carencia en el desarrollo y la comprensión de las relaciones, es decir, las dificultades para adaptarse a los diferentes contextos como hacer amigos, compartir juegos imaginativos, por la falta de interés que se tiene de sus compañeros.

4. Tienden a patrones repetitivos y restringidos de comportamientos, intereses y actividades.

5. Obsesión por algún tema en concreto de interés, con profunda intensidad o enfoque.

Los síntomas están presentes en el periodo de desarrollo temprano, pero existen casos que no se manifiestan por estar enmascarados por estrategias aprendidas en la edad adulta. Suelen causar un deterioro clínicamente significativo en áreas sociales ocupacionales. Si bien, dadas las características de este estudio, se ocupará de los niños con SA.

Generalmente, los niños con este diagnóstico tienen limitaciones para entender y emplear las reglas de conducta social que se suman a los déficits de habilidades sociales adecuadas y a la carencia de intuición para determinar cuándo utilizar esas habilidades (Bauminger, 2003). Además, presentan dificultades para comprender y expresar emociones, así como para interpretar emociones y expresiones faciales (Gutstein \& Whitney, 2002; Shaked \& Yirmiya, 2003; Lindner \& Rosén, 2006).

Los déficits sociales muestran repercusiones en las interacciones con la familia, compañeros y otros adultos, y también interfieren en su desarrollo normal, académico, emocional y social (Krasny, Williams, Provenzal \& Ozonoff, 2003; Rao et al., 2008). Vázquez y Murillo (2007) definen a los niños con SA como:

Según Simpson (2004), en un artículo sobre una guía para los padres de niños con SA, los familiares de los niños con SA comentan que se están enfrentando diariamente a una serie de retos para los cuales tienen muy poca información. Experimentan decepciones, frustraciones y toda una variedad de emociones relacionadas directa o indirectamente con el trastorno. Una de las mayores preocupaciones que presentan los padres de estos niños es que sus hijos no interactúan bien con los demás niños. Es común que los padres comenten que su hijo casi nunca está "presente en el momento", que es muy poco consciente de la gente, los objetos y los acontecimientos que ocurren en el momento y que su mente está en otro lugar (pensando activamente), pero no aquí y ahora.

Gresham, Sugai y Horner (2001) afirman que la habilidad de interactuar exitosamente con los pares y adultos significativos es una de las áreas más importantes del desarrollo de los alumnos.

Una máxima que se hacen la mayoría de los terapeutas que trabajan con niños con SA es ¿qué es lo que podemos hacer para asistir a estos niños a comprender su entorno social y ser exitosos navegando en nuestro complejo mundo social? Así pues, principalmente se necesita dirigir su aprendizaje social y emocional enseñándoles las habilidades esenciales para desarrollar competencias sociales y emocionales. Esto incluye el 
entrenamiento de habilidades sociales en las siguientes áreas: resolución de problemas, habilidades de conversación, identificación de sentimientos y emociones, manejo de las emociones y los sentimientos, control de la ira, manejarse con estrés y las habilidades de organización.

Es fundamental que los terapeutas y familias enseñen y refuercen habilidades pragmáticas a los niños con SA, necesitando practicarlas en entornos distintos y en múltiples situaciones para conseguir crear conciencia pragmática en su habla.

La conciencia pragmática hace referencia a la capacidad autorreflexiva y crítica que tienen los hablantes para someter a juicio el uso que hacen de su propia lengua. Esto es, la autovaloración de sus destrezas y habilidades lingüístico-discursivas y, en definitiva, de su comportamiento comunicativo cuando utilizan el lenguaje en sus prácticas sociales. Otra forma de definirla, según Reyes (2002), sería la conciencia sobre qué formas del lenguaje son apropiadas al contexto de comunicación, siendo una habilidad para darse cuenta de que uno como hablante $u$ otras personas hacen un uso apropiado o no del lenguaje, ya que una expresión usada en el contexto familiar puede no ser apropiada tanto para el lenguaje escrito como para la comunicación con otras personas que no sean la propia familia.

Algunos autores van aún más lejos y afirman que la pragmática «se ha convertido en un depósito de todo tipo de consideraciones extragramaticales y de los efectos de esos factores en la forma gramatical y léxica» (Horn, 1990, p.45).

Aprovechando algunas de las contribuciones procedentes de la antropología lingüística, destacan las propuestas de Silverstein (1993), para quien la actividad metalingüística se fundamenta en la metapragmática; esto es, un proceso reflexivo más general que tiene que ver con la funcionalidad comunicativa de los enunciados y sus condiciones de uso. En otras palabras, la competencia metapragmática se refiere al conocimiento que tiene el propio usuario de las relaciones existentes dentro del sistema y entre el sistema lingüístico y el contexto comunicativo en el que se produce. Por lo tanto, esta competencia es la que permite al enunciador distinguir el mensaje lingüístico del contexto extralingüístico y establecer conexiones entre ambos.

En las interacciones orales se activa la competencia pragmática y, por lo tanto, constituyen un punto de referencia clave para describir y explicar tanto el dominio como los desórdenes comunicativos.

Gallardo (2006), en uno de sus artículos, propone que el componente pragmático está dividido en tres grandes dimensiones:

Pragmática enunciativa, categoría que surge por la consideración de cada enunciado como una acción intencional por parte del hablante.

Pragmática textual, categoría vinculada a la naturaleza gramatical del mensaje que construye el hablante, y que para tener tal valor de mensaje debe tener cohesión y coherencia.

Pragmática interactiva, categoría pragmática que surge al considerar que todo mensaje se dirige a un receptor, por lo que se centra en la toma de turno conversacional. 
Teniendo presente que uno de los principales objetivos que se propone un profesional de la logopedia es trabajar mediante una intervención directa con los niños con TA, dirigida a mejorar la comunicación, incrementar la socialización y desarrollar sus habilidades sociales, combinando normalmente estrategias conductuales y educativas (Jané, Ballespí, \& Doménech-Llabería, 2006; Corsi Sliminng, Guerra, \& Plaza, 2007).

El entrenamiento en habilidades sociales implica la enseñanza de habilidades mediante la aplicación de técnicas conductuales y de aprendizaje social: modelado, ensayos conductuales, feedback, representación de papeles y ensayos en vivo (Cooper, Griffith, \& Filer, 1999; Klin \& Volkmar, 2000). En algunos programas de habilidades sociales se incluyen actividades dirigidas a mejorar la compresión socioemocional, el reconocimiento y la expresión de emociones (Attwood, 2000; Martín-Borreguero, 2004; Olivar \& de la Iglesia, 2008); un claro ejemplo de actividad para ello sería "La teoría de la mente".

Actualmente existe una grandísima carencia de información sobre estudios realizados acerca de la conciencia pragmática en niños con SA, lo cual es una motivación para elaborar algo novedoso que llevará a tener diferentes puntos de vista de cómo el niño, la familia y el terapeuta que conviven y trabajan con él son conscientes de las competencias pragmáticas del propio niño SA. Por todo ello, el objetivo de este estudio ha sido realizar una comparación de la conciencia pragmática en función de la perspectiva de niños con Asperger, sus familias y los terapeutas que le tratan.

\section{MARCO EMPÍRICO}

\section{Objetivo general}

Conocer la conciencia pragmática de los alumnos con SA según ellos mismos, sus familias y los terapeutas.

Objetivos específicos:

- Establecer la conciencia que tiene el propio niño con SA sobre su conciencia pragmática propiamente dicha, precisando la visión de la familia y el terapeuta. Contrastar las perspectivas acerca de la conciencia pragmática que tienen los alumnos con SA, sus familias y terapeutas que les atienden.

\section{DISEÑO}

Esta investigación tiene un planteamiento metodológico cuantitativo de carácter descriptivo-comparativo, en cuanto que pretende conocer la percepción que tienen los niños con SA, sus familias y terapeutas, tratando de hacer también una comparación entre ellas. Desde este planteamiento, se trata de una investigación de tipo descriptiva donde el propósito citado se logra mediante la cumplimentación de un cuestionario de elaboración propia. 


\section{PARTICIPANTES}

Los participantes han sido seleccionados de manera no aleatoria debido al bajo índice de prevalencia que presenta el SA (Fombonne, 2005). Para formar la muestra se tuvo en cuenta un único criterio de inclusión, ser niño con SA, familiar o terapeuta de este. No se consideraron criterios de exclusión. Se han seleccionado los participantes mediante muestreo intencional no aleatorio. En la totalidad de los casos, los niños que componen la muestra, aparte del apoyo familiar, asisten a las asociaciones de Asperger, y actualmente reciben intervención logopédica y pedagógica, trabajando con mayor importancia el entrenamiento en habilidades socioemocionales.

El cuestionario se le ha administrado a un total de 18 personas, (véase tabla 1), entre ellas:

\begin{tabular}{lccc}
\hline & & $\delta$ & क \\
\hline \multirow{2}{*}{ Alumnos } & $\mathrm{F}$ & 7 & 1 \\
& $\%$ & 87.5 & 12.5 \\
\hline \multirow{2}{*}{ Familiares } & $\mathrm{F}$ & 1 & 7 \\
& $\%$ & 12.5 & 87.5 \\
\hline \multirow{2}{*}{ Terapeutas } & $\mathrm{F}$ & 0 & 2 \\
& $\%$ & 0 & 100 \\
\hline
\end{tabular}

Tabla 1.

Distribución de los participantes por colectivo

Fuente: Los autores

\section{Edades}

\begin{tabular}{llllllll}
\cline { 2 - 7 } & 6 & 7 & 8 & 9 & 10 & 11 & 12 \\
\hline No Alumnos & 3 & 2 & 1 & 0 & 1 & 1 & 0 \\
\hline
\end{tabular}

Tabla 2.

Distribución de los alumnos con SA por edades

Fuente: Los autores

\section{INSTRUMENTOS}

El instrumento utilizado para el desarrollo de este estudio ha sido un cuestionario ad hoc diseñado tras realizar una revisión de documental y no encontrar ningún instrumento que midiera específicamente la conciencia pragmática en niños con síndrome de Asperger.

El instrumento consta de tres versiones diferentes en función de los destinatarios por los que iba a ser cumplimentado: terapeutas, niños con SA y familiares de niños con SA. Solo ha variado la forma de redacción 
en función de a quién fuese dirigido y quien lo cumplimentara, por ello se elaboraron tres versiones, aunque conteniendo las mismas preguntas.

En todos ellos se evalúan cuatro dimensiones de información de tipo sociodemográfico: edad, curso, género y parentesco. El resto del cuestionario consta de 30 ítems, distribuidos como variables ordinales, siguiendo una escala tipo Likert de 4 puntos, donde $1=$ nunca, $2=$ a veces, $3=$ a menudo y $4=$ siempre, excepto el último, que permite añadir observaciones.

El Cuestionario de Conciencia Pragmática para niños con SA (CCPNSA) definitivo se recoge en la tabla 3.

\begin{tabular}{|c|c|c|}
\hline \multicolumn{3}{|c|}{ VERSIÓN FINAL DE LOS CUESTIONARIOS } \\
\hline Bloque & Dimensiones & Ítems \\
\hline Acciones en el habla & Tipo de habla, los gestos, expresividad, miradas. & $1-8$ \\
\hline Cercanía al comunicar & $\begin{array}{l}\text { Cercanía y contacto físico a la persona, forma de } \\
\text { dirigirse a las personas. }\end{array}$ & $9-12$ \\
\hline $\begin{array}{l}\text { Comprensión de } \\
\text { Conversaciones }\end{array}$ & $\begin{array}{l}\text { Comprensión, relación de los temas, adaptación } \\
\text { de las conversaciones. }\end{array}$ & $13-17$ \\
\hline Confianza en el habla & $\begin{array}{l}\text { Utilización de refuerzos para llegar a resolver los } \\
\text { problemas de comprensión. }\end{array}$ & $18-21$ \\
\hline Pautas de conversación & $\begin{array}{l}\text { Respeto de los turnos en las conversaciones y la } \\
\text { agilidad en dichas relaciones comunicativas. }\end{array}$ & $22-27$ \\
\hline Construcción del lenguaje & $\begin{array}{l}\text { Estructura del lenguaje y relación de las } \\
\text { conversaciones. }\end{array}$ & $28-30$ \\
\hline
\end{tabular}

Tabla 3.

Distribución de ítems por dimensiones

Fuente: Los autores

\section{VALIDEZ}

Tras un proceso de revisión exhaustiva de los cuestionarios, se procedió a validación por juicio de expertos. Para ello, se les envió los cuestionarios a tres expertos: una logopeda, una maestra de audición y lenguaje y un orientador. Se valoró la consistencia interna de cada uno de los ítems, concretamente la claridad y la relevancia de cada ítem, siguiendo a Escobar y Cuervo (2007). Puntúa 1 si lo consideraban nada claro/relevante, 2 si lo consideraban poco claro/relevante, 3 claro/relevante y 4 muy claro/ relevante. Se volvió a revisar teniendo en cuenta esta vez las valoraciones de los expertos en cada ítem, cuya media sea igual o inferior a tres puntos en claridad o en relevancia.

Por supuesto, se tuvieron presentes las sugerencias de los expertos en algunas preguntas y a partir de ahí se conformó la versión final de los cuestionarios: terapeutas, niños con SA y familiares. 


\section{Cuestionario Alfa de Cronbach $\mathrm{N}^{\circ}$ de ítems}

\begin{tabular}{lll}
\hline Alumnos & .874 & 30 \\
Familiares & .914 & 30 \\
Terapeutas & .877 & 30 \\
\hline
\end{tabular}

\section{Tabla 4 \\ Análisis de fiabilidad \\ Fuente: Los autores}

En la evaluación de los coeficientes de alfa de Cronbach que hace De Vellis (2003), y basándonos en esta clasificación (tabla 4), los cuestionarios obtienen una fiabilidad respetable (entre .80 y .90).

\section{PROCEDIMIENTO}

Esta investigación comenzó llevando a cabo una revisión bibliográfica de artículos relacionados con la conciencia pragmática en niños con SA. Más adelante se llevó a cabo el diseño de los tres cuestionarios de recogida de información que llevó a varias revisiones exhaustivas. Para valorarlo se procedió a un juicio de expertos, como se ha indicado en el apartado instrumentos.

La aplicación de los cuestionarios se llevó a cabo en un gabinete de logopedia y en un centro educativo, siendo administrados en formato digital. Los cuestionarios de los alumnos y terapeuta fueron cumplimentados en presencia de la investigadora, excepto el de los padres que lo hicieron en solitario. Dicho cuestionario realizado a través de la aplicación formularios Google, elegido por la rapidez y facilidad del uso, y además una resolución completa de los resultados, donde por cada pregunta mostraba una gráfica con la frecuencia y el porcentaje de respuesta. La aplicación se realizó en una misma semana, en el horario de trabajo logopédico y pedagógico, tanto a los niños, las familias y los terapeutas.

Finalmente, se procedió al vaciado y análisis de datos en el BM SPSS Statistics (vers. 19) y se han elaborado las conclusiones y la redacción del presente informe.

\section{ANÁLISIS DE DATOS}

El análisis de datos se llevó a cabo con el programa IBM SPSS Statistics (vers. 19). Para determinar las frecuencias de cada una de las variables, se optó por un análisis descriptivo por medio de tablas de contingencia y se calculó la validez mediante el coeficiente alfa de Cronbach. Por último, dadas las características de la muestra, para resolver la existencia 
de diferencias significativas entre grupos se utilizó test no paramétrico de Kruskal-Wallis.

\section{RESULTADOS}

A continuación, se presenta un análisis de resultados atendiendo a cada uno de los objetivos.

Objetivo 1. Establecer la conciencia que tiene el propio niño con $S A$ sobre su pragmática propiamente dicha, precisando la visión de la familia y determinando el punto de vista que tiene el terapeuta.

\begin{tabular}{|c|c|c|c|}
\hline & Niños & Familia & Terapeuta \\
\hline & N AV AM S & N AV AM S & N AV AM S \\
\hline 1.Tu ritmo al hablar es el adecuado & 02223 & 0242 & 1420 \\
\hline 2. Hablas en voz alta y con adecuada entonación & 03332 & 0422 & 0661 \\
\hline 3.Realizas movimientos de brazos y manos al hablar & 24220 & 04440 & 0530 \\
\hline 4.Cuando hablas cambias de posición los pies & 2510 & 04440 & 3320 \\
\hline 5.Acompañas las conversaciones con gestos faciales & $3 \begin{array}{lll}3 & 1 & 1\end{array}$ & 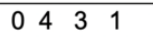 & 4400 \\
\hline 6.Miras a la cara con quien estás hablando & 02224 & 23330 & 3311 \\
\hline 7.Te interesa lo que hagan o piensen los demás & 1241 & 14330 & 51111 \\
\hline 8. Aceptas las críticas de los demás & $4 \begin{array}{lll}4 & 0 & 1\end{array}$ & 22222 & $\begin{array}{llll}3 & 4 & 0 & 1\end{array}$ \\
\hline
\end{tabular}

Tabla 5.

Bloque Acciones al hablar.

Fuente: Los autores

Los resultados (véase tabla 5), se muestran muy similares desde las tres perspectivas, si bien es destacable en el ítem 3, realizas movimientos de brazos y manos al hablar, observando que hay niños que comentan que nunca (f.2), sin embargo, la familia (f.4) y el terapeuta (f.5), destacan mayormente que a veces.

Los datos ponen de manifiesto que los niños dicen que miran a la cara cuando hablan con otras personas, sin embargo, los datos de familias y terapeutas indican que no es así. Los niños responden que siempre miran a la cara (f.4), cosa que la familia y el terapeuta recalcan que a veces o a menudo (f.3).

Por último, observamos que tanto los niños (f.4) como las familias (f.4) dicen que solo muestran interés a menudo o a veces hacia lo que los demás hacen o piensan, mientras que el terapeuta (f.5) opina que nunca. 


\begin{tabular}{|c|c|c|c|}
\hline & Niños & Familia & Terapeuta \\
\hline & N AV AM S & N AV AM S & N AV AM S \\
\hline 9. Cuando hablas tu cara expresa tu estado de ánimo & 1600 & 0152 & 4310 \\
\hline 10. Te gusta acercarte a las personas cuando hablas & 2402 & 0620 & 0800 \\
\hline 11. Utilizas el contacto físico al hablar & 43300 & 2420 & 4310 \\
\hline 12. Llamas a las personas por su nombre & 11106 & 12332 & 3320 \\
\hline
\end{tabular}

Tabla 6.

Bloque Cercanía al comunicar.

Fuente: Los autores

Los datos (véase tabla 6), nos hacen destacar el ítem 9, cuando hablas tu cara expresa tu estado de ánimo, que nos muestra que los niños (f.6) piensan que su cara expresa su estado de ánimo, a diferencia por ejemplo del terapeuta (f.4) que destacan que nunca.

Según lo observado, los niños (f.6) opinan que la hora de dirigirse a alguien siempre la llaman por su nombre, sin embargo, la familia y el terapeuta no opinan igual.

\begin{tabular}{|c|c|c|c|}
\hline & Niño & Familia & Terapeuta \\
\hline & N AV AM S & N AV AM S & N AV AM S \\
\hline 13. Cuando hablas tus amigos entienden el mensaje & 04440 & 0512 & 0143 \\
\hline 14. Te ríes con los chistes que cuentan los demás & 0312 & 0350 & 2330 \\
\hline 15. Te adaptas a los cambios de tema al conversar & 05512 & 0215 & 0413 \\
\hline 16. Relacionas las ideas en una conversación & 06610 & 05521 & 1331 \\
\hline 17. Te cuesta entender los comentarios irónicos & 1223 & 1520 & 2330 \\
\hline
\end{tabular}

Tabla 7.

Bloque Comprensión de conversaciones

Fuente: Los autores

Los datos (véase tabla 7), manifiestan que la familia (f.2) y las terapeutas (f.3) consideran que algunos niños cuando hablan sus amigos sí que entienden el mensaje, en cambio los resultados de los propios niños (f.4) revelan que solo a veces se sienten entendidos.

En cuanto al ítem 17, te cuesta entender los comentarios irónicos, se encontró que solo algunos niños (f.3) nos confirman que siempre les es difícil comprender las ironías de los demás, al contrario de las familias (f.5), que opinan que solo a veces.

\begin{tabular}{|c|c|c|c|}
\hline & Niños & Familia & Terapeuta \\
\hline & N AV AM S & N AV AMS & NAVAM S \\
\hline 18. Cuando lo necesitas pides ayuda a los demás & 0431 & 1340 & 2330 \\
\hline 19. Te adaptas a los cambios de tema al conversar & 0412 & 2330 & 0710 \\
\hline 20. Relacionas las ideas en una conversación & 1412 & 1340 & 0710 \\
\hline 21. Te cuesta entender los comentarios irónicos & 2222 & 23221 & 0413 \\
\hline
\end{tabular}

Tabla 8.

Bloque Confianza en el habla

Fuente: Los autores 
Los resultados obtenidos (véase tabla 8), hacen destacar el ítem 19, preguntas a los demás cuando tienes dudas, donde las terapeutas (f.7) responden que la mayoría de los niños solo a veces preguntan cuando tienen dudas, a diferencia de las familias (f.2) que opinan que nunca.

De igual forma ocurre en el siguiente ítem, donde el terapeuta (f.7) destaca que la mayoría de los niños solo a veces se comunican de diferente forma, ya estén hablando con sus padres, amigos, profesores, a diferencia de las familias (f.4), que contestan que solo algunos niños a menudo cambian el registro según con quien estén hablando.

\begin{tabular}{|c|c|c|c|}
\hline & Niños & Familia & Terapeuta \\
\hline & N AV AM S & N AV AM S & N AV AM S \\
\hline 22. Respetas tu turno en las conversaciones & $\begin{array}{llll}0 & 0 & 3 & 5\end{array}$ & 22222 & 1700 \\
\hline 23. Te molesta que te interrumpan cuando hablas & 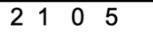 & $\begin{array}{llll}0 & 3 & 4 & 1\end{array}$ & 0521 \\
\hline 24. Interrumpes cuando los demás están hablando & 1610 & 1412 & 1520 \\
\hline 25. Cuando te preguntan respondes rápido & 05521 & 03350 & 1700 \\
\hline 26. Te gusta dar información en tus conversaciones & 2222 & 02224 & 0422 \\
\hline 27.. Aportas ideas nuevas cuando hablas & 2240 & 1430 & 2510 \\
\hline
\end{tabular}

Tabla 9.

Bloque Pautas de comunicación

Fuente: Los autores

Los resultados extraídos (véase tabla 9), muestra cómo los niños (f.5) opinan que respetan siempre el turno en las conversaciones en conjunto, sin embargo, las terapeutas (f.7) responden que la mayoría de los niños solo lo hacen a veces.

Se resalta el ítem 23, te molesta que te interrumpan cuando hablas, donde se observa que los propios niños (f.5) responden que les molesta que los interrumpan cuando están hablando, en cambio tanto la familia como los terapeutas (f.0), coinciden en la opinión de que los niños no se muestran molestos cuando los interrumpen.

\begin{tabular}{|c|c|c|c|}
\hline & Niños & Familia & Terapeuta \\
\hline & $\mathrm{N}$ AV AM S & N AV AM S & N AV AM S \\
\hline 28. Construyes frases sin cometer muchos errores & 13222 & $\begin{array}{llll}0 & 4 & 1 & 3\end{array}$ & 2510 \\
\hline 29. Empleas diferentes palabras para decir lo mismo & $\begin{array}{llll}3 & 3 & 2 & 0\end{array}$ & 06620 & 2312 \\
\hline 30.. Relacionas fácilmente las diferentes ideas & $\begin{array}{llll}1 & 4 & 3 & 0\end{array}$ & 13313 & 5300 \\
\hline
\end{tabular}

Tabla 10.

Bloque Construcciones en el habla.

Fuente: Los autores

A partir de los resultados obtenidos (véase tabla 10), se observa cómo la mayoría de las madres (f.6) consideran que sus hijos suelen emplear a veces sinónimos para referirse a lo misma palabra, en cambio tanto el niño (f.3) como las terapeutas (f.2) contestan en algunos casos que los niños nunca emplean sinónimos.

Y, por último, se puede manifestar cómo las terapeutas (f.5) en la mayoría de los casos opinan que los niños nunca relacionan fácilmente las distintas ideas de una conversación, cosa que los niños (f.4. y madres (f.3) 
dicen que, según de lo que se trate la conversación, a veces relacionan bien las conversaciones y otras no.

Objetivo 2. Contrastar las perspectivas acerca de la conciencia pragmática que tienen los alumnos con $S A$, sus familias y terapeutas que les atienden.

Para dar respuesta a este objetivo y contrastar si existen diferencias estadísticamente significativas entre las percepciones que tienen los niños con SA, sus familiares y terapeutas han llevado a cabo la prueba $\mathrm{H}$ de Kruskal-Wallis.

Tras realizar la prueba se ha verificado que existe un efecto estadísticamente significativo solamente en dos de los ítems evaluados: ítem 9, cuando habla un niño con $S A$ su cara suele expresar su estado de ánimo $(\mathrm{H}=9705, \mathrm{gl}=1, \mathrm{p}=.002)$ e ítem 22, los niños con $S A$ respetan su turno en las conversaciones con variaspersonas $(\mathrm{H}=11850$, $\mathrm{gl} .=2, \mathrm{p}=.003)$.

En el resto de los ítems, la prueba $\mathrm{H}$ de Kruskal-Wallis, permite evidenciar que no existe ningún efecto estadísticamente significativo, con lo cual se afirma que las precepciones que tienen de la conciencia pragmática de los niños con SA los propios niños, sus familiares y los terapeutas son muy parecidas.

\section{DISCUSIÓN Y CONCLUSIONES}

Tras analizar los resultados obtenidos en este estudio, se observa que hay varios de los aspectos recogidos en el marco teórico de la investigación que son confirmados por medio de los resultados antes expuestos.

En primer lugar, tanto los niños con SA, sus familias como los terapeutas coinciden que en que tienen problemas de comunicación verbal y no verbal y de relación con los demás, coincidiendo con el DSM-5 (2014). Ello queda patente en acciones que facilitan la conversación verbal como no verbal, como son movimientos de manos y pies, acompañar las conversaciones con gestos faciales, aceptar las críticas de los demás, utilizar el contacto físico al hablar, expresar el estado de ánimo, donde los tres grupos coinciden en que lo suelen hacer a veces.

Resulta relevante que, según la percepción de las familias, los niños con SA a menudo son cercanos al comunicar, si bien los niños y terapeutas estiman que esa cercanía es solo a veces.

Finalmente, en esta investigación se ha observado que la percepción que tienen los familiares, niños con SA y familiares responden a las características definidas para este tipo de niños tal cual las identifican Krasny, Williams, Provenzal y Ozonoff, (2003); Vázquez y Murillo (2007); Rao et al., (2008). Son niños con buena gramática, vocabulario rico, pero conscientes de sus limitaciones en la comunicación no verbal.

Los resultados de este estudio deben considerarse en relación con una serie de limitaciones, que a continuación se enumeran: 
- Ausencia de instrumentos que midiesen la conciencia pragmática del niño con SA a partir de la perspectiva del propio niño, su familia y su terapeuta.

- Dificultad para realizar una validación más exhaustiva del instrumento diseñado, puesto que solamente ha sido posible realizarla por medio del juicio de expertos.

- A la hora de pasar dicho cuestionario al niño, se han encontrado con bastantes problemas de interpretación, por lo que han necesitado en todo momento una ayuda para leérselo y formulárselo para que no diesen contestaciones sin saber qué pedía dicha pregunta.

- Otra de las restricciones relevantes es la referida al tamaño de la muestra: ocho niños diagnosticados con SA limitan el poder estadístico y la capacidad de generalizar los resultados obtenidos.

\section{REFERENCIAS}

1. American Psychiatric Association. (2000). Diagnostic and statistical manual of mental disorders. Washington, DC.

2. American Psychiatric Association. (2014). DSM-5. Manual diagnóstico y estadístico de los trastornos mentales. Editorial Médica Panamericana.

3. Asperger, H. (1944). Die "Autistischen Psychopathen" im Kindesalter. Archiv für Psychiatrie und Nervenkrankheiten. 117(1), 76-136.

4. Attwood, T. (2000). Strategies for improving the social integration of children with Asperger Syndrome. Autism, 4, 85-100.

5. Bauminger, N. (2003). Peer interaction and loneliness in high functioning children with autism. Journal of Autism and Developmental Disorders, (33), 489-507.

6. Bauminger, N., \& Kasari, C. (2003). Loneliness and Friendship in HighFunctioning Children with Autism. Child Development, 71(2), 447-456.

7. Cooper, M., Griffith, K., \& Filer, J. (1999). School intervention for inclusion of students with and without disabilities. Focus on Autism and Other Developmental Disabilities, (14), 110-115.

8. De la Iglesia, M., \& Olivar, J. (2008). Intervenciones socio comunicativas en los trastornos del espectro autista de alto funcionamiento. Revista de Psicopatologia y Psicología Clínica, 13, 1-19.

9. DeVellis, R. (2003). Scale development: Theory and applications. Thousand Oaks, CA: Sage.

10. Escobar-Pérez, J., \& Cuervo-Martínez, A. (2008). Validez de contenido y juicio de expertos: una aproximación a su utilización. Avances en Medición, (6), 27-36. Recuperado de:

11. http://www.humanas.unal.edu.co/psicometria/files/7113/8574/5708/Art iculo3_Juicio_de_expertos_27-36.pdf

12. Fombonne, E. (2003). Epidemiological surveys of autism and other pervasive developmental disorders: an update. Journal of Autism and Developmental Disorders, 33, 365-382.

13. Frances, A., First, M., \& Pincus, H. (2002). DSM-IV-TR: Manual de diagnóstico diferencial. Editorial Masson. 
14. Gallardo, B. (2006). Más allá de las palabras y su estructura: las categorías del componente pragmático. En Garayzábal E. (Coord.). Lingüistica clínica y logopedia (p. 81---196). Madrid: Machado Libros.

15. Gresham, F., Sugai, G., \& Horner, R. (2001). Interpreting outcomes of social skills training for students with high-incidence disabilities. Exceptional Children, 67(3), 331-344.

16. Gutstein, S., \& Whitney, T. (2002). Asperger syndrome and the development of social competence. Focus on Autism and Other Developmental Disabilities, 17(3), 161-171.

17. Horn, L. (1990). Teoría pragmática. En F. Newmeyer (Ed.), Panorama de la lingüistica moderna de la Universidad de Cambridge. Madrid: Visor.

18. Jané, M. Ballespí, S., \& Doménech-Llabería, E. (2006). Un caso de trastorno de Asperger. En Méndez, F., Espada, J., \& Orgilés, M. (dirs.), Intervención psicológica y educativa con niños y adolescentes. Madrid: Pirámide.

19. Klin, A., \& Volkmar, F. (2000). Treatment and intervention guidelines for ind viduals with Asperger's Syndrome. En Klin, A., Volkmar, F., \& Sparrow, S. (dirs.), Asperger syndrome. Nueva York: Guilford.

20. Krasny, L., Williams, B., Provencal, S., \& Ozonoff, S. (2003). Social skills interventions for the autism spectrum: essential ingredients and a model curriculum. Child and Adolescent Psychiatric Clinics in North America, 12, 107-122.

21. Lindner, J., \& Rosén, L. (2006). Decoding of emotion through facial expression, prosody and verbal content in children and adolescent with Asperger's syndrome. Journal of Autism and Developmental Disorders, 36, 769-777.

22. Martín-Borreguero, P. (2005). Perfil lingüístico del individuo con síndrome de Asperger: implicaciones para la investigación y la práctica clínica. Revista de neurología, 41(1), 115-122.

23. Silverstein, M. (1993). Metapragmatic discourse and metapragmatic function. En Lucy, J. (Ed.), Reflexive language. Cambridge: Cambridge University Press.

24. Simpson, R. (2004). Finding effective intervention and personnel preparation practices for students with autism spectrum disorders. Exceptional Children, (70), 135-144.

25. Shaked, M., \& Yirmiya, N. (2003). Understanding social difficulties. En Prior, M. (dirs.), Learning and behavior problems in Asperger syndrome. Nueva York: Guilford.

26. Rao, P., Beidel, D., \& Murray, J. (2008). Social skills interventions for children with Asperger's syndrome or high-functioning autism: a review and recommendations. Journal of Autism and Developmental Disorders, (38), 353-361.

27. Reyes, G. (2002). Metapragmática. Lenguaje sobre lenguaje, ficciones, figuras. Valladolid: Universidad de Valladolid.

28. Vásquez, B., \& del Sol, M. (2017). Características Neuroanatómicas del Síndrome de Asperger. International Journal of Morphology, 35(1), 376-385.

29. Wing, L. \& Gould, J. (1979). Severe impairments of social interaction and associated abnormalities in children: epidemiology and classification. Journal of Autism and Developmental Disor.ers, 9(1), 11-29. 
30. Williams, S., Koenig, K., \& Scahill, L. (2007). Social skills development in children with autism spectrum disorders: A review of the intervention research. Journal of Autism and Developmental Disorders, 37(10), 1858-1868. 